\title{
Role of vitamin D in the development and prognosis of carcinoma-insight of treatment strategy in Pakistan.
}

\author{
Sarrah Ali*, Laila tul Qadar, Osama Shabbir \\ Dow University of Health Sciences, Karachi, Sindh, Pakistan
}

Accepted on July 19, 2018

Vitamin D deficiency (VDD) is the most prevalent, undiagnosed and mal-treated nutritional deficiency worldwide [1]. Pakistan, a South Asian country located near the equator is the victim of VDD which is common in every age and both genders despite being sunbathed the whole year. This is clearly a mystery waiting to be solved. A recent study conducted by Mubashir et al. discovered that about $75.7 \%$ of Pakistani population is predominantly VD deficient [2]. The best source of Vitamin D (VD) is sunlight Ultraviolet B rays. It is absorbed by melanocytes present in the epidermal layer of human skin which is then converted into its most active form 1,25 dihydroxycholecalciferol by liver and kidneys respectively. Studies have shown possible reasons for Pakistani population being hypovitaminosed-D is either due to darker pigmentation of skin hindering the synthesis of VD or addictive habit of chewing betel-nuts thus leading to VDD by altering the enzymes regulating circulating $1,25(\mathrm{OH})(2) \mathrm{D}$ concentration $[3,4]$.

$\mathrm{VD}$ is a steroid hormone regulating many cellular pathways through its gene induced transcription effects. Many epidemiological studies have shown a positive association with good prognosis of cancer and VD supplementation, although it is not tested on human models but rectifying VDD can decrease metastatic incidences. In its active form, calcitriol supplementation is effectively shown to enhance ALA-PDT chemotherapeutic effect on breast cancer cells in a tumor effective manner with least damage to skin [5]. It is an attractive approach for maximal patient's comfort. VDD amplifies the growth of BCa (Breast Carcinoma) as these cells express VDR (Vitamin D receptor) which regulates gene transcription of IDI oncogene associated with breast cancer [6]. These studies suggest that the people with low circulating level of VD are at a significantly higher risk of developing cancer and correcting its deficiency can protect mass population.

The discrepancy between health practitioners of Pakistan and recommended treatment of VDD is worth mentioning. Patients are prescribed with high and prolonged doses of VD supplements for joints pain and weakness without knowing their whole history opens a new chapter of health problems. People thoughtlessly following such prescriptions can face toxicity due to increased absorption of Zinc, Calcium and Lead resulting in nausea, vomiting, stomach pain, constipation, bone loss, hypercalciuria, kidney failure, fatigue and dizziness etc. [7].

It is concluded that VDD is a strong predisposing factor of cancer development, which can alarmingly affecting most of the Pakistan's population. More studies should be carried out on human model to confirm the role of VD in tumor suppression.
It will help to significantly reduce the cost of treatments as well as enhance patient's comfort and compliance to chemotherapy. However, blind prescription is not a solution, but a malpractice. It is suggested that circulating calcitriol levels should be tested before the commencement of VD supplementation [8]. Moreover in order to effectively eradicate VDD from society, it is necessary to educate masses about VD prevailing deficiency and its consequences especially in regard to development of Breast Cancer.

\section{References}

1. Gupta A. Vitamin D deficiency in India: prevalence, causalities and interventions. Nutrients. 2014 Feb 21;6(2):729-75.

2. Mubashir M, Anwar S, Tareen AK, et al. Association of Vitamin D deficiency and VDBP gene polymorphism with the risk of AMI in a Pakistani population. Pakistan journal of medical sciences. 2017 Nov;33(6):1349.

3. Ogunkolade WB, Boucher BJ, Bustin SA, et al. Vitamin $\mathrm{D}$ metabolism in peripheral blood mononuclear cells is influenced by chewing "betel nut"(Areca catechu) and vitamin D status. The Journal of Clinical Endocrinology \& Metabolism. 2006 Jul 1;91(7):2612-7.

4. Masood SH, Iqbal MP. Prevalence of vitamin D deficiency in South Asia. angiogenesis. 2008 Oct 1;1(11):12.

5. Rollakanti KR, Anand S, Maytin EV. Vitamin D enhances the efficacy of photodynamic therapy in a murine model of breast cancer. Cancer medicine. 2015 May;4(5):633-42.

6. Williams JD, Aggarwal A, Swami S, et al. Tumor autonomous effects of vitamin D deficiency promote breast cancer metastasis. Endocrinology. 2016 Mar 2;157(4):1341-7.

7. Kemp FW, Neti PV, Howell RW, et al. Elevated blood lead concentrations and vitamin D deficiency in winter and summer in young urban children. Environmental health perspectives. $2007 \mathrm{Apr} ; 115(4): 630$.

8. Kennel KA, Drake MT, Hurley DL. Vitamin D deficiency in adults: when to test and how to treat. InMayo Clinic Proceedings 2010 Aug 1 (Vol. 85, No. 8, pp. 752-758). Elsevier.

\section{*Correspondence to:}

\section{Ali S}

Dow University of Health Sciences

Karachi, Sindh, Pakistan

E-mail: sarrahali97@gmail.com 